http://jmscr.igmpublication.org/home/ ISSN (e)-2347-176x ISSN (p) 2455-0450 crossref DOI: https://dx.doi.org/10.18535/jmscr/v8i3.63

\title{
Left Ventricular Global Longitudinal Strain Following Revascularization in Acute ST Elevation Myocardial Infarction-A Comparison of Primary Angioplasty and Fibrinolytic Therapy
}

\author{
Authors \\ Dr Vechalapu Ravindra Dev, Dr D. Rajasekhar, Dr V. Vanajakshamma \\ Department of Cardiology, Sri Venkateswara Institute of Medical Sciences, Tirupati, Andhra Pradesh, \\ 517502, India
}

\begin{abstract}
This is a single centre, prospective and observational study that compares the efficacy of fibrinolytic therapy followed by elective revascularisation versus primary PCI in patients with STEMI by using global longitudinal LV strain.

Keywords: Primary PCI, fibrinolytic therapy, STEMI, Global longitudinal LV strain.
\end{abstract}

\section{Introduction}

Myocardial infarction is clinically diagnosed by symptoms of myocardial ischemia combined with evidence of myocardial infarction on electrocardiographic, biochemical or imaging modalities. The practical approach to a patient with symptoms of myocardial ischemia is to consider them into a syndrome called acute coronary syndrome (ACS). ACS includes the diagnoses of ST elevation myocardial infarction (STEMI), non-ST elevation myocardial infarction (NSTEMI) and unstable angina. The important investigation needed to differentiate the patients of ACS is a 12-lead ECG, by which we can differentiate the patients with ST elevation or without ST elevation ${ }^{[1]}$

In the past three decades, the mortality rate for patients with STEMI had shown a steady fall which stabilized in the last 10 years. This decline is due to both decrease in the incidence of STEMI and decrease in mortality rate of patients who presented with STEMI due to better management options. The management options for STEMI includes reperfusion by either catheter-based reperfusion techniques or by giving a fibrinolytic agent like streptokinase, reteplase or tenecteplase. Of both these management strategies, primary PCI is the preferred option for reperfusion, if the patient arrives at a facility which is capable of performing primary PCI. But, in India, most of the hospitals do not have the facilities to perform primary PCI. If the time taken for the first medical contact to perform primary PCI is expected to take more than 120 minutes, it is indicated to administer a fibrinolytic agent in a patient with STEMI who presented within 12 hours of onset of chest pain and with no contraindications for fibrinolysis. In addition to this, even if the time for transfer of the patient to PCIcapable facility is short, administration of a fibrinolytic agent immediately is advantageous if the patient presented very early and without any contraindications. If PCI is used as an initial reperfusion therapy in STEMI patients, it is called 
as primary PCI. If the PCI is performed after a failed fibrinolysis, it is called rescue $\mathrm{PCI}^{[2,3]}$.

In echocardiography, the term 'strain' is used to describe local shortening, thickening and lengthening of the myocardium as a measure of regional Left Ventricular function ${ }^{[1,2]}$. This myocardial strain can be used as a principle for measuring the left ventricular systolic function. LV strain can be calculated for each segment in radial, longitudinal and circumferential. GLS which is measured in longitudinal direction and reflects the longitudinal contraction of the myocardium is the best evaluated parameter of all strain parameters. GLS is a more sensitive parameter than LV ejection fraction while measuring the LV systolic function and GLS can also be used to identify subclinical LV dysfunction. Apart from measuring the LV systolic function, GLS also has other uses. The risk of ventricular arrhythmias can be identified by intersegmental variability in timing of peak myocardial strain. GLS strain imaging is also helpful for guiding the position of the LV pacing lead in patients who undergo cardiac resynchronisation therapy.

Global Longitudinal Strain (GLS) is a recently developed technique for detecting the early stages of LV systolic dysfunction ${ }^{[2,3]}$. Global longitudinal strain measures the myocardial movement in the longitudinal direction. GLS has less inter-observer variability and it gives more consistent results than LV ejection fraction.

Imaging modalities used for measuring global longitudinal strain are tissue Doppler imaging (TDI) or by speckle-tracking echocardiography (STE) ${ }^{[3,4]}$. Tissue Doppler imaging is an older technique which has several drawbacks like angle dependency. Now, speckle tracking echocardiography is the most widely used imaging modality for measuring GLS. Speckles are the markers which are equally distributed throughout the myocardium. The motion of these tracking speckles during the LV contraction are analysed by the speckle tracking echocardiography (STE) using the ultrasonic images of the LV myocardium during systole in two dimensions. The movement of the speckles represents contraction of that particular area of the myocardium during systole. By tracking these speckles, myocardial strain can be calculated. 2D speckle tracking echocardiography is angle independent as it tracks the movement of speckles along the direction of the wall and not in the direction of the ultrasound beam ${ }^{[5]}$. Thus, it is more accurate than Tissue Doppler imaging.

\section{Materials and Methods}

This is a single centre, prospective, comparative and observational study conducted in the department of Cardiology, in a tertiary care teaching institute from January to March 2019. The study is carried out after clearance by the Institutional Thesis Approval and Ethics Committees.

A written informed consent form is obtained from all patients participating in the study.

\section{Inclusion Criteria}

All patients with acute ST elevation myocardial infarction who presented to the department of Cardiology with chest pain and underwent primary PCI or received fibrinolytic therapy followed by elective revascularisation if indicated are included in the study. Patients who present with STEMI underwent primary PCI or fibrinolytic therapy and the choice is left to the individual treating clinician. In patients who underwent primary PCI, pre discharge global longitudinal strain (GLS) is calculated. Patients who received fibrinolytic therapy underwent subsequent angiogram and angioplasty if required. Global longitudinal strain is calculated pre discharge.

\section{Exclusion Criteria}

1) History suggestive of previous MI or ECG changes suggestive of previous MI.

2) Previous reports suggestive of LV systolic dysfunction or historical evidence of heart failure symptoms.

3) Patients with renal dysfunction.

4) Pregnant patients.

5) Patients with any contraindications for fibrinolytics or antiplatelets.

6) Patients with underlying malignancies.

7) Patients who underwent CABG. 
8) Unwilling patients.

Cardiovascular risk factor profile including history of diabetes, hypertension, smoking, dyslipidemia, family history of coronary artery disease is collected. Vitals, cardiovascular examination findings and blood reports are collected. After the PCI, the LV systolic function is measured by using LVEF and GLS, which is the primary outcome.

After the angioplasty, the LV systolic function was measured before discharge of the patient by using GLS via speckle tracking echocardiography and LVEF by using Simpson's method. Global longitudinal strain (GLS) was estimated by 2D STE using the SIEMENS ACUSONSSEC2000 following the guidelines of journal of American society of echocardiography.

\section{Results}

The present study included 120 patients with Acute STEMI and satisfying the inclusion and exclusion criteria, who presented to the department of Cardiology. There were 60 patients in the primary PCI arm and 60 patients in the fibrinolysis followed by elective revascularisation arm.

Table 1. Primary Outcome

\begin{tabular}{|c|c|c|c|}
$\begin{array}{c}\text { Primary } \\
\text { outcome }\end{array}$ & $\begin{array}{c}\text { Primary } \\
\mathbf{n = 6 0}\end{array}$ & $\begin{array}{c}\text { Fibrinolytic } \\
(\mathbf{n}=60)\end{array}$ & $\begin{array}{c}\mathbf{p}- \\
\text { value }\end{array}$ \\
\hline GLS(\%) & $-11.5 \%$ & $-9.5 \%$ & $0.03^{*}$ \\
\hline LVEF(\%) & $45.6 \%$ & $41.2 \%$ & $0.02^{*}$ \\
\hline
\end{tabular}

$>$ GLS- global longitudinal strain, LVEF- left ventricular ejection fraction.

$>*$ indicates significant $\mathrm{p}$-value.

The mean age was $54 \pm 13$ years (range: $31-78$ years) in the study population. The mean age in the primary PCI group was $53 \pm 13$ years and $57 \pm 11$ years in the fibrinolysis followed by elective revascularisation group. (p-value: 0.13 ).

Global longitudinal strain measured pre-discharge was significantly better in patients of STEMI who underwent primary PCI than in patients who underwent fibrinolysis followed by elective revascularisation indicating better outcomes with primary PCI.

\section{Conclusion}

The myocardial salvage, and thereby the post MI LV function indicated by global longitudinal strain following fibrinolysis followed by elective revascularisation strategy in acute ST elevation myocardial infarction, fares inferior to primary angioplasty. Hence, primary angioplasty should be the revascularisation strategy of choice wherever feasible. Fibrinolysis followed by elective revascularisation strategy should be reserved only to areas where primary PCI could not be performed.

\section{Discussion}

STEMI leads to transmural necrosis of the myocardium and the amount of myocardium necrosed will determine the LV systolic function after the STEMI. PCI leads to improvement in blood flow to myocardium which can restore the hibernating myocardium. This amount of restored myocardium will ultimately determine the LV systolic function after angioplasty. If more amounts of hibernating myocardium is restored to its normal function, higher will be the LV systolic function which ultimately leads to better outcomes.

In this study, GLS was better in patients who underwent primary PCI than who underwent fibrinolysis followed by elective revascularisation. This shows that post revascularisation LV function was better with primary PCI, which ultimately leads to better outcomes. This may be due to better myocardial salvage with primary PCI than fibrinolysis

\section{References}

1. Falk E, Nakano M, Bentzon J, Finn A, Virmani R. Update on acute coronary syndromes: the pathologists' view, European Heart Journal. 2012;34(10):719-728.

2. Perk G, Tunick P, Kronzon I. Non-Doppler Two-dimensional Strain Imaging by Echocardiography-From Technical Considerations to Clinical Applications, Journal of the American Society of Echocardiography. 2007;20(3):234-243. 
3. Gilman G, Khandheria B, Hagen M, Abraham T, Seward J, Belohlavek M. Strain rate and strain: A step-by-step approach to image and data acquisition, Journal of the American Society of Echocardiography. 2004;17(9):1011-1020.

4. Heimdal A, Støylen A, Torp H, Skjærpe T. Real-Time Strain Rate Imaging of the Left Ventricle by Ultrasound. Journal of the American Society of Echocardiography. 1998;11(11):1013-1019.

5. Dandel M, Knosalla C, Lehmkuhl H, Hetzer R. Non-Doppler two-dimensional strain imaging - clinical application, Journal of the American Society of Echocardiography. 2007;20(8):1119. 